\title{
Agiles Lernen digital gestützt: Die Methode eduScrum in der Hochschullehre
}

\section{Nico Sturm und Heike Rundnagel}

\section{Zusammenfassung}

Der Beitrag geht, im Rahmen einer explorativen Vorgehensweise, den Fragen nach, inwieweit der Einsatz der digital angereicherten Methode eduScrum in der Hochschullehre den Kompetenzerwerb bei Studierenden sowohl in Bezug auf fachliche und personale als auch im Hinblick auf digitale Kompetenzen fördert. Hierzu werden im Rahmen von Veranstaltungsevaluationen vorgenommene Selbsteinschätzungen der Studierenden über den eigenen Kompetenzzuwachs herangezogen. Als Bezugspunkte für die in der Evaluation erstellten Fragen zur Einschätzung der Kompetenzentwicklung und zur Einordnung der aus diesen Betrachtungen generierten Ergebnisse dienen der „Deutsche Qualifikationsrahmen für Lebenslanges Lernen“ (DQR), der „,Qualifikationsrahmen für deutsche Hochschulabschlüsse“ (HQR) und das „Digital Competence Framework for Citizens" (DigComp 2.1).

\section{Schlüsselwörter}

Agilität • Agiles Lernen • Hochschullehre • Selbststeuerung • EduScrum • Evaluation • Kompetenzen

N. Sturm (凶)

Stadt Neu-Anspach, Deutschland

E-Mail: nico.sturm@neu-anspach.de

H. Rundnagel

Philipps-Universität Marburg, Marburg, Deutschland

E-Mail: heike.rundnagel@ verwaltung.uni-marburg.de 


\section{Hochschullehre agil und digital gestalten}

Hochschulen erfüllen, aus unterschiedlichen Perspektiven heraus betrachtet, eine wichtige Rolle. Metzner et al. folgend leisten die Hochschulen in einer systemischen Perspektive ,gleichzeitig einen wichtigen Beitrag zur Innovations- und Wandlungsfähigkeit von Wissenschaft, Gesellschaft und Volkswirtschaft" (2019, S. 11). Indem sie auf der einen Seite Wissen weiterentwickeln und festigen und auf der anderen Seite ihre Absolventinnen und Absolventen ,zur Übernahme komplexer wissenschaftlicher Tätigkeiten [befähigen]“ (ebenda).

Entsprechend dieser Feststellung des Diskussionspapiers ,Was bedeutet Hochschullehre im digitalen Zeitalter?", aber auch vieler weiterer Überlegungen zur Hochschullehre in der heutigen globalisierten, digitalisierten Wissensgesellschaft wird deutlich, dass Hochschullehrende in der Gestaltung von Lehr-/ Lernarrangements einer Vielzahl von Anforderungen entsprechen müssen, wenn sie den vielfältigen, teils unterschiedlichen Erwartungen gerecht werden wollen. Der Deutsche Qualifikationsrahmen für Lebenslanges Lernen (DQR) definiert, dass sowohl Fachkompetenzen (Wissen und Fertigkeiten) als auch personale Kompetenzen (Sozialkompetenz und Selbstständigkeit) auf akademischem Niveau gezielt zu vermitteln sind (Arbeitskreis DQR 2011, S. 5). Gleichzeitig gewinnt der sichere und kompetente Umgang mit digitalen Medien im Alltag und in der Hochschullehre immer mehr an Bedeutung (Meyer-Guckel et al. 2019, S. 4).

Mit Blick auf die Anforderungen an hochschulische Lehre, wie beispielsweise durch heterogene Studierendenkohorten mit unterschiedlichen Bedarfen und Bedürfnissen an die zeitliche und räumliche Gestaltung von Lehr-/ Lernarrangements, aber auch auf die Erwartungen eines sich wandelnden Arbeitsmarktes gilt es, Absolvent*innen im Sinne einer späteren Beschäftigungsfähigkeit mit entsprechenden Kompetenzen auszustatten. Darüber hinaus wird der Bedarf an digitalen Lehr-/ Lernarrangements, die in der Hochschullehre zu einem effektiven Kompetenzerwerb im Sinne der Lernzielerreichung beitragen, durch zusätzliche Herausforderungen katalysiert.

Die Verfassenden haben sich dieser Herausforderungen angenommen und gehen im vorliegenden Beitrag - am Beispiel der digital angereicherten agilen Methode ,eduScrum“ (eduScrum: digital) - der folgenden Frage nach: Kann der Einsatz dieser Methode die Entwicklung von fachlichen und personalen Kompetenzen im Sinne der Lernziele eines Studienprogramms als auch die gezielte Entwicklung digitaler Kompetenzen fördern? Um sich dieser Fragestellung anzunähern, wird ein quantitativ angelegtes Vorgehen mit evaluativem Charakter herangezogen, welches auf der Grundlage der Referenzrahmen ,Deutsche Qualifikationsrahmen für Lebenslanges Lernen“ (DQR), „Qualifikationsrahmen für 
deutsche Hochschulabschlüsse“ (HQR) und des „Digital Competence Framework for Citizens“ (DigComp 2.1) erarbeitet wurde. $\mathrm{Zu}$ diesem Zweck wurden die Studierenden zweier Seminare der Erziehungs- und Bildungswissenschaften, welche mit der Methode eduScrum: digital durchgeführt wurden, unter anderem zur Einschätzung der eigenen Kompetenzentwicklung befragt.

In einem ersten Schritt werden zunächst die Methode eduScrum in ihren Grundzügen dargestellt und die digitale Anreicherung und Umsetzung im Rahmen der untersuchten Lehr-/ Lernarrangements beschrieben (2). Danach werden unter Rückgriff auf die Referenzrahmen Bezugspunkte für die Bestimmung der zu erreichenden Kompetenzen in ausgewählten Kompetenzbereichen, die im weiteren Verlauf vertiefend betrachtet werden, ausgeführt (3). In einem dritten Schritt wird das Forschungsvorgehen, welches zur Beantwortung der in diesem Beitrag formulierten Forschungsfrage herangezogen wurde, erörtert (4). Im vierten und letzten Schritt werden die Ergebnisse in Bezug auf die gewählten Referenzrahmen präsentiert, eingeordnet und in einer abschließenden Kurzzusammenfassung auf die Forschungsfrage zurückgebunden (5).

\section{2 eduScrum: digital umgesetzt}

Seit dem Sommersemester 2019 setzt Nico Sturm die Methode in Seminaren des Bachelorstudiengangs Erziehungs- und Bildungswissenschaften an der PhilippsUniversität Marburg ein. Zum Wintersemester 2019/20 wurde das Konzept digital angereichert: Es wurde auf die Lernplattform der Hochschule überführt, integriert und in zwei parallel laufenden Seminaren im gleichen Bereich umgesetzt. ${ }^{1}$ Im Folgenden werden sowohl die zentralen Aspekte der Methode eduScrum im Allgemeinen als auch der digitalen Umsetzung im Speziellen komprimiert dargelegt.

\subsection{Die Methode eduScrum}

eduScrum ist ein auf Lehr-/ Lernarrangements adaptiertes Rahmenwerk, das auf der agilen Methode Scrum basiert, die ursprünglich entwickelt wurde, um komplexe IT-Projekte effektiv organisieren und bearbeiten zu können (vgl. Schwaber

\footnotetext{
${ }^{1}$ Das Projekt wurde im Rahmen eines Fellowships vom Projekt ,Zukunftswerkstatt für digital gestütztes Lehren und Lernen“ der Philipps-Universität Marburg https://uni-marburg.de/ vMnbX begleitet und unterstützt.
} 
und Sutherland 2017, S. 3). Wie auch in Scrum arbeiten in eduScrum Teams von jeweils drei bis fünf Personen (Lernende) zusammen. Sie werden von der Lehrperson in die Methode eingeführt und bekommen die zu erreichenden Lernergebnisse sowie die für die Zielerreichung zur Verfügung stehende Zeit vorgegeben. Davon ausgehend bestimmen die Teams ihren Lernweg zur Erreichung der vorgegebenen Lernziele komplett eigenständig. Durch die drei Grundsätze von eduScrum: Transparenz, Überprüfbarkeit und Anpassung, wird dieser Prozess methodisch strukturiert.

Durch Transparenz wird sichergestellt, dass die wesentlichen Aspekte des Prozesses für alle Akteur*innen (Teammitglieder und Lehrperson) ständig aktuell und sichtbar sind. Hierfür ist es unter anderem notwendig, ein gemeinsames Verständnis von den Lernzielen sowie von den für die Zielerreichung zu erledigenden Aufgaben zu entwickeln.

Ein Effekt dieser methodisch indizierten Transparenz ist die Überprüfbarkeit des jeweils aktuellen Arbeitsstandes sowie der Qualität des bisher Geleisteten. So wird es möglich, unerwünschte Abweichungen frühzeitig zu erkennen und potenzielle Risiken für eine rechtzeitige Zielerreichung zu kontrollieren. Bei der Überprüfbarkeit geht es explizit nicht um eine Bewertung durch die Lehrperson. Vielmehr bringen Überprüfungen den größten Nutzen, wenn sie von den eduScrum-Teams eigenverantwortlich und regelmäßig selbst vorgenommen werden. Die Lehrperson wird lediglich bei Unterstützungsbedarf hinzugezogen.

Vollständige Transparenz und Überprüfbarkeit des Lernprozesses bilden die Basis für die Möglichkeit von Anpassungen. Die eduScrum-Teams haben jederzeit die Möglichkeit, ihren Lernweg und ihr Vorgehen anzupassen. Der wesentliche Vorteil besteht darin, dass gar nicht erst mit großem Aufwand ,unerwünschte“ Ergebnisse erzeugt werden. Vielmehr kann prozessbegleitend nachjustiert werden.

Um die drei Grundsätze methodisch zu operationalisieren, den Arbeitsprozess zu strukturieren und eine Regelmäßigkeit sowie Vorhersehbarkeit zu gewährleisten, verwendet eduScrum fest definierte und wiederkehrende Ereignisse (Zusammenkünfte der Teams, in denen Transparenz und Überprüfbarkeit hergestellt wird und Anpassungen vorgenommen werden). Zusammengefasst basiert das Konzept auf der Transparenz sämtlicher Prozesse, der fortlaufenden Überprüfbarkeit des Lernfortschrittes sowie der Möglichkeit der ständigen Veränderung und Anpassung des Lernweges mit dem Ziel, die von der Lehrperson vorgegebenen Lernergebnisse in der zur Verfügung stehenden Zeit zu erreichen (vgl. Wijnands

\footnotetext{
${ }^{2}$ eduScrum orientiert sich sehr eng an der ursprünglich für die Organisation komplexer Projekte entwickelten Methode Scrum. Es wurde jedoch eine Anpassung an den jeweiligen Bildungskontext vorgenommen (wie beispielsweise Namen der unterschiedlichen Rollen), um einen einfachen Zugang zur Methode für die Lernenden zu ermöglichen.
} 
und Stolze 2019, S. 106 f.; Delhij et al. 2015). ${ }^{3}$ Aus der Sicht der Verfassenden wird diese Methode damit den eingangs formulierten Anforderungen an eine moderne universitäre Ausbildung in hohem Maße gerecht.

\subsection{Die digital angereicherte Umsetzung der Methode}

Für eine entsprechende digitale Umsetzung der Methode wurden die Grundlagen der Methode sowie die zentralen Arbeitsformen in die Lernplattform der Hochschule - ILIAS ${ }^{4}$ überführt. Hierzu wurden für das jeweilige Seminar auf der Lernplattform zwei Ebenen eingezogen: die Kursebene und die Gruppenebene.

Die Kursebene wurde so ausgestaltet, dass sie den Studierenden die Einarbeitung in die Methode und deren Arbeitsweisen erleichtern und die Kontaktaufnahme mit anderen Studierenden und der Lehrperson ermöglichen sollte. Dort wurden zudem Möglichkeiten der Selbstüberprüfung der Lernzielerreichung sowie Gruppenräume bereitgestellt. Auf der Kursebene wurden

1. sämtliche Informationen zur Methode eduScrum in einem sehr umfangreichen Wiki bereitgestellt, welches Verlinkungen und erklärende Grafiken enthält,

2. Informationen über die technischen Möglichkeiten der Lernplattform in Form von Texten und Erklärvideos eingestellt; dort wurden die ILIAS-Tools und ihre Anwendung beschrieben, die:

a) zum Zusammentragen, zum Organisieren und Aufbereiten gemeinsam erarbeiteter Wissensbestände geeignet sind (Etherpad, Blog, Wiki, Forum, Datei, Ordner, Test),

b) eine direkte oder auch zeitlich und räumlich entkoppelte Kommunikation miteinander ermöglichen (Chat, Forum, Etherpad, Videokonferenz),

3. zusätzlich Möglichkeiten geschaffen, bei technischen oder inhaltlichen Fragen und Problemen mit der Lehrperson in Kontakt zu treten (Forum, Buchungspool zur Organisation der [virtuellen] Sprechstunde),

${ }^{3}$ Für eine ausführliche Darstellung der Methode eduScrum siehe Delhij et al. (2015, S. 5 ff.). Für eine breiter angelegte Diskussion zu den Potenzialen von eduScrum für die Hochschullehre siehe Sturm und Rundnagel (2020).

${ }^{4}$ ILIAS ist die zentrale, onlinegestützte Lehr-Lern-Plattform der Philipps-Universität Marburg, die auf den Servern der Hochschule gehostet wird. Weitere Informationen unter anderem unter: https://uni-marburg.de/gcYXB. Eine Adaptierung (der hier exemplarisch in ILIAS umgesetzten Methode) auf anderen Plattformen (Moodle, StudIP et cetera) ist durchaus möglich. Die komplette Dokumentation, Ansicht des erstellten Kurses sowie Datei zur Weiterverwendung findet sich hier: https://uni-marburg.de/cS6Bk. 
4. die Lernziele des gesamten Kurses entlang der einzelnen Themenbereiche bereitgestellt, durch Basistexte ergänzt und durch Selbstüberprüfungstests die Möglichkeit für die Studierenden geschaffen, den eigenen Stand mit Blick auf die Lernziele zu überprüfen (lernzielorientierter Kurs, Datei, Test).

Darüber hinaus wurden auf Kursebene Zugänge zu (geschlossenen) Gruppenräumen geschaffen, in denen die Studierenden ihre Teamarbeit und ihren Lernprozess eigenverantwortlich gestalten, miteinander kommunizieren und ihre Arbeitsergebnisse erstellen können. In diesen Gruppenräumen erhielten die Studierenden sämtliche Schreibrechte und konnten alle Anwendungen, die die Lernplattform zur Verfügung stellt, erproben und nutzen. Auf diese Weise wurde den Studierenden der größtmögliche Gestaltungsfreiraum auf dieser Oberfläche verschafft. Kernstück dieser Gruppenräume waren:

1. Textfelder, in denen die Studierenden die im Team getroffenen Verabredungen zur Arbeit im Team, zur Fertigstellung der Aufgaben und zur ständigen Selbstüberprüfung bearbeiten konnten (Seiteneditor),

2. eine Datensammlung, in der sämtliche Aufgaben des Teams und seiner Mitglieder mit Zuständigkeiten und insbesondere dem Stand der Fertigstellung aufgeführt waren (Datensammlung),

3. alle Anwendungen, deren Verwendung auf der Kursebene beschrieben wurden, um ihre Kooperation und Erstellung der gemeinsamen Produkte zu verabreden und gemeinsam zu erarbeiten (Etherpad, Blog, Wiki, Forum, Datei, Ordner, Test, Chat, Videokonferenz).

\section{Die Referenzrahmen}

Da es sich bei dem vorliegenden Anwendungsfall um Lehrveranstaltungen handelt, die im Rahmen der akademischen Ausbildung auf Bachelorniveau angeboten werden, bietet sich zum Einordnen der (weiter) zu entwickelnden Kompetenzen der Deutsche Qualifikationsrahmen für Lebenslanges Lernen (DQR) mit Bezug auf fachliche und personale Kompetenzen - ergänzt durch den Qualifikationsrahmen für deutsche Hochschulabschlüsse (HQR) und das Digital Competence Framework for Citizens (DigComp 2.1) mit Blick auf digitale Kompetenzen besonders an. Ein weiteres gewichtiges Argument für die Auswahl dieser Referenzrahmen besteht darin, dass sie den Europäischen Qualifikationsrahmen für Lebenslanges Lernen (EQR/EQF) als gemeinsamen Ausgangspunkt haben. Entsprechend dem EQR lassen sich daher Kompetenzen ableiten, die für die 
Erlangung des Bachelorniveaus - DQR 6 und DigComp 2.1 5/ 6 - in diesem festgelegt wurden.

Die Referenzrahmen dienten der Bestimmung der zu erreichenden Lernergebnisse und $\mathrm{zu}$ entwickelnden Kompetenzen im Rahmen der Entwicklung der Methode eduScrum: digital. Gleichzeitig werden die ausgewählten Lernziele herangezogen, um eine Einschätzung abgeben zu können, inwiefern durch den Einsatz der Methode eine Weiterentwicklung der Kompetenzen erfolgt ist. Im Folgenden werden daher diese Referenzrahmen skizziert und die für das digital angereicherte Konzept von eduScrum ausgewählten Kompetenzbereiche herausgearbeitet. ${ }^{5}$

\subsection{DQR/HQR}

Der DQR ist ein Referenzrahmen, der bildungsbereichsübergreifend alle Qualifikationen des deutschen Bildungssystems erfasst. Er beschreibt auf insgesamt acht Niveaustufen Kompetenzen, die für die Erlangung der jeweiligen Qualifikation erforderlich sind (vgl. Arbeitskreis DQR 2011, S. 4). Auf jeder Niveaustufe wird zwischen zwei Kompetenzkategorien (Fachkompetenz und personale Kompetenz) unterschieden. Diese werden weiter ausdifferenziert, indem die Kompetenzkategorie „Fachkompetenz“ in „Wissen“ und „Fertigkeiten“ und die Kompetenzkategorie „personale Kompetenz“ in "Sozialkompetenz“ und „Selbstständigkeit“ geteilt wird. Vor dem Hintergrund des zu analysierenden Umsetzungsszenarios zeigt Tab. 1 die für die Erlangung des Bachelorniveaus formulierten Kompetenzen.

Da sich das vorliegende Erkenntnisinteresse explizit auf den Erwerb fachunabhängiger Kompetenzen bezieht, bleibt die Kategorie „Wissen“ bei der Überprüfung des Kompetenzzuwachses im Folgenden unberïcksichtigt.

Um den Kompetenzzuwachs der Studierenden bei der Durchführung der Methode eduScrum: digital noch etwas feingliedriger in den Blick nehmen zu können, wurde zusätzlich der HQR herangezogen (Hochschulrektorenkonferenz, Kultusministerkonferenz 2017). Dieser greift die Niveaustufen 6 (Bachelorniveau), 7 (Masterniveau) und 8 (Doktoratsebene) aus dem DQR auf und differenziert die Kompetenzprofile akademischer Bildung auf diesen drei Stufen weiter aus. Dazu differenziert er auf jeder Stufe zwischen „Wissen und Verstehen“ und „Können (Wissenserschließung)“. Während die Kategorie „Wissen und

\footnotetext{
${ }^{5}$ Da der vorliegende Beitrag den Anspruch formuliert, der Frage nachzugehen, ob eduScrum: digital studienfachunabhängig den Kompetenzerwerb unterstützt, fokussieren sich die Betrachtungen auf die überfachlichen Kompetenzen der Referenzrahmen. Alle fachbezogenen inhaltlichen Lernziele bleiben dabei unberücksichtigt.
} 


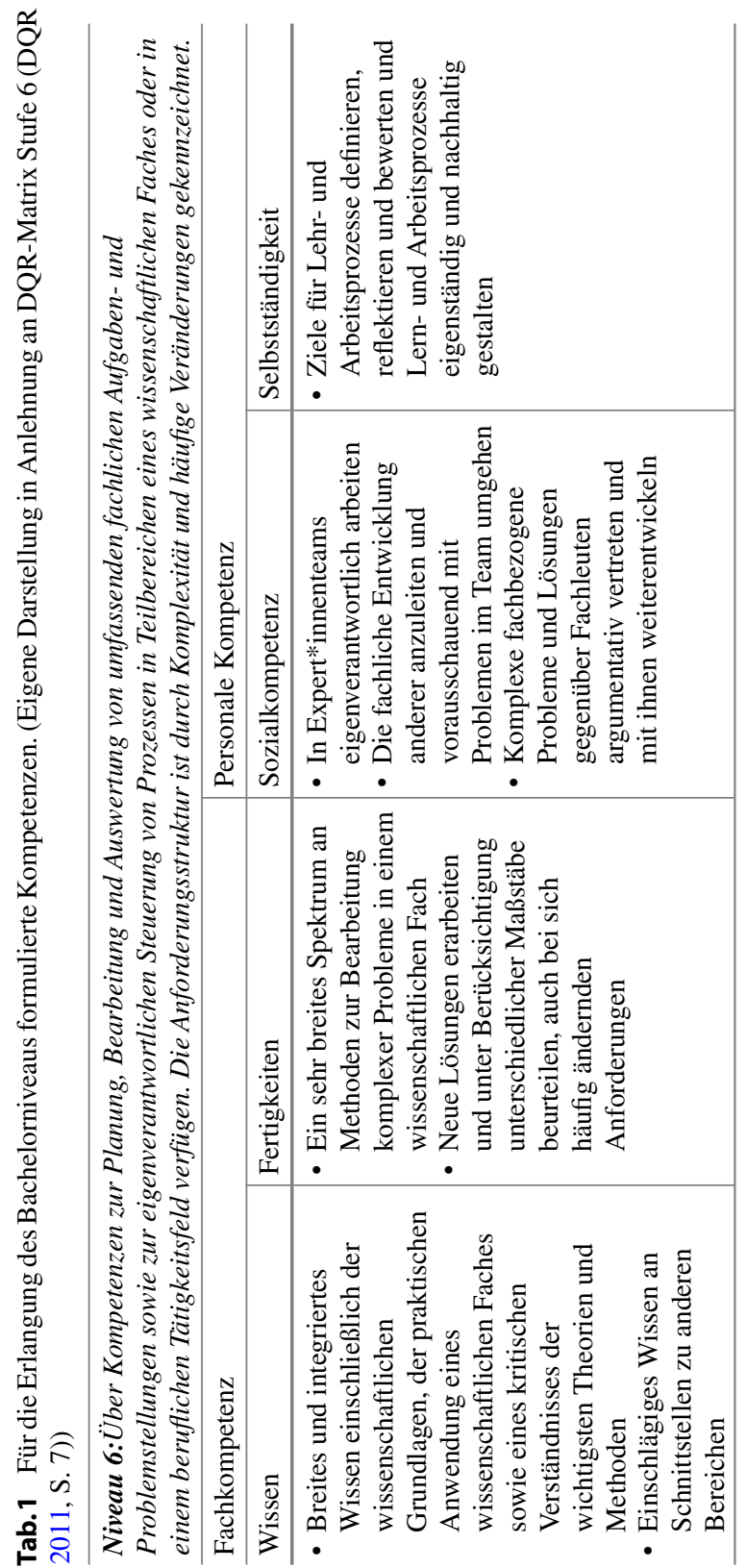


Verstehen“ Kompetenzen mit dem Blick auf den fachspezifischen Wissenserwerb (Fachkompetenzen) beschreibt, zielt die Kategorie „Können (Wissenserschließung)“ auf Kompetenzen ab, die dazu befähigen, ,Wissen anzuwenden und einen Wissenstransfer zu leisten“ (DQR 2011, S. 13). Diese auf die Beschreibung von Methodenkompetenz ausgerichtete Kategorie berücksichtigt auch kommunikative und soziale Kompetenzen und hat eine fachunabhängige Gültigkeit.

Mit Verweis auf die Zielsetzung des Beitrages wird die weitere Betrachtung - wie auch schon beim DQR - auf die Kompetenzen fokussiert, die mit dem erfolgreichen Abschluss eines Bachelorstudiums erworben werden sollen, unabhängig davon, welches Fach studiert wurde. Daher werden - wie in Tab. 2 dargestellt - ausschließlich die Kriterien der Kategorie „Können“ in die weiteren Betrachtungen einbezogen. 


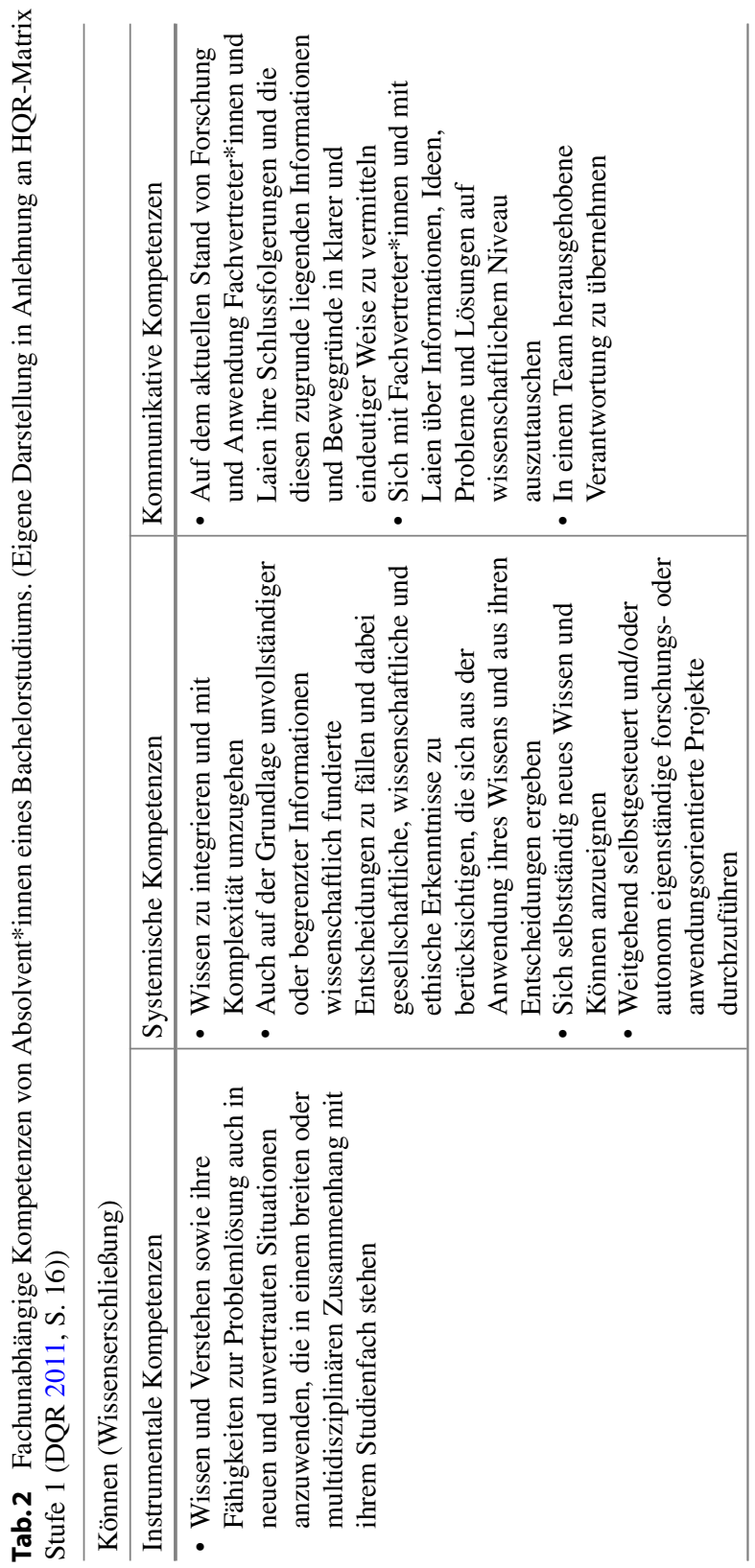




\subsection{DigComp 2.1}

Der DigComp 2.1 ist eine Weiterentwicklung des „Digital Competence Framework for Citizens“ (Ferrari et al. 2013, 2014) und des überarbeiteten „DigComp 2.0“ (Vuorikari et al. 2016) und wurde vom Joint Research Center (JRC) der Europäischen Union veröffentlicht. Ziel des Rahmenwerks ist die Bereitstellung eines Werkzeugs zur Verbesserung digitaler Kompetenzen von Bürger*innen (vgl. Carretero et al. 2017, S. 7).

Der DigComp 2.1 unterscheidet fünf Dimensionen: Informations- und Datenkompetenz, Kommunikation und Kollaboration, Erstellen digitaler Inhalte, Sicherheit sowie Problemlösen und differenziert diese auf acht Qualifikationslevel - abgeleitet aus dem EQR und damit äquivalent zum DQR - aus. Näher definiert werden die Qualifikationslevel, genau wie im DQR, in Bezug auf die Komplexität der gestellten Aufgabe, die Autonomie der Aufgabenbearbeitung sowie der kognitiven Lernziele, welche den Bloomschen Taxonomiestufen entsprechen (Carretero et al. 2017, S. 12 f.). Wie beschrieben wird das fortgeschrittene Niveau (Stufen 5/6) als Bezugspunkt für die weiteren Betrachtungen herangezogen.

Für das Vorgehen haben die Verfassenden die in Tab. 3 dargestellten Kompetenzbereiche aufgrund ihres speziellen Fokus der Methode auf das selbst gesteuerte, kollaborative Arbeiten sowie das Produzieren digitaler Arbeitsergebnisse als besonders aufschlussreich angenommen, da diese die Operationalisierung der Methode eduScrum in den Seminaren am konkretesten abbilden. Grundlegend war die Annahme, dass die Besonderheiten der Methode (siehe Abschn. 2.1) in

Tab. 3 Ausgewählte Kompetenzbereiche des DigComp 2.1. (Eigene Darstellung nach DigComp 2.1 (Carretero et al. 2017, S. 26 ff.))

\begin{tabular}{|c|c|c|}
\hline \multicolumn{3}{|l|}{ Kompetenzbereich } \\
\hline $\begin{array}{l}\text { Informations- und } \\
\text { Datenkompetenz }\end{array}$ & Kommunikation und Kollaboration & Digitale Inhalte erstellen \\
\hline $\begin{array}{l}\text { Suchen und Filtern von } \\
\text { Daten, Informationen } \\
\text { und digitalen Inhalten } \\
\text { - Bewerten von Daten, } \\
\text { Informationen und } \\
\text { digitalen Inhalten } \\
\text { Verwalten von Daten, } \\
\text { Informationen und } \\
\text { digitalen Inhalten }\end{array}$ & $\begin{array}{l}\text { - Interagieren durch digitale } \\
\text { Technologien } \\
\text { - Teilen durch digitale Technologien } \\
\text { - Bürgerengagement } \\
\text { (Verantwortungsübernahme) durch } \\
\text { digitale Technologien } \\
\text { - Zusammenarbeiten durch digitale } \\
\text { Technologien } \\
\text { - Kommunikationsstandards } \\
\text { - Verwalten von digitalen Identitäten }\end{array}$ & $\begin{array}{l}\text { - Entwickeln von digitalen } \\
\text { Inhalten } \\
\text { - Einbinden und } \\
\text { Neubearbeitung von } \\
\text { digitalen Inhalten } \\
\text { - Urheberrecht und } \\
\text { Lizenzen } \\
\text { - Programmieren }\end{array}$ \\
\hline
\end{tabular}


der digitalen Umsetzung stärker ausgeprägt zur Geltung kommen und die Studierenden insbesondere in diesen Bereichen ihre Kompetenz im Umgang mit Medien (weiter)entwickeln können.

In dieser von den Verfassenden aus den Inhalten des DigComp 2.1 selbst erstellten Tabelle wird die Ausdifferenzierung, die der Referenzrahmen für die drei gewählten Kompetenzbereiche vornimmt, dargestellt. Diese bilden den Ausgangspunkt für die exemplarische Überprüfung der Kompetenzentwicklung durch den Einsatz von eduScrum: digital. Im Folgenden soll nun das Forschungsvorgehen beschrieben werden.

\section{$4 \quad$ Rahmende Forschung}

Zur Überprüfung der Kompetenzentwicklung von Studierenden in Bezug auf die vorgestellten Referenzrahmen (1.3) griffen die Verfassenden auf eine Kombination von quantitativen und qualitativen Methoden zurück. ${ }^{6}$ Der vorliegende Beitrag fokussiert dabei jedoch auf die Ergebnisse der quantitativen Fragebogenerhebungen.

In den untersuchten Seminaren „Bildungsmanagement“ (BM) und „Kompetenzen in der Erwachsenenbildung" (Komp) wurden sowohl Eingangs- als auch Ausgangsbefragungen durchgeführt. Alle Befragungen wurden digital in ILIAS umgesetzt. Die Teilnahme an der Eingangsbefragung wurde an die Aufnahme in den ILIAS-Kurs geknüpft und war daher für alle Teilnehmenden verpflichtend (BM $\mathrm{n}=19$; Komp $\mathrm{n}=50$ ). Die Teilnahme an der Ausgangsbefragung war eingeschränkt freiwillig, da sie an die Abgabe der Studienleistung im Seminar geknüpft wurde (BM n = 17; Komp $n=40)$. Insgesamt haben an beiden Kursen 66 von 69 angemeldeten Studierenden teilgenommen, die in der Regel im dritten oder fünften Semester studieren.

In der Befragung wurden sowohl offene Fragen als auch Matrixfragen mit Ranking-Skalen (vgl. Diekmann 2018, S. 471 f.) verwendet. Hierbei wurden die aus den Referenzrahmen (HQR, DQR, DigComp 2.1) abgeleiteten Kompetenzen

\footnotetext{
${ }^{6}$ Die Befragungen wurden ergänzt durch das Erheben von Reflexionsportfolios und teilnehmender Beobachtung. Ziel einer solchen Erhebung war es, die Ergebnisse zur gegenseitigen Validierung (vgl. Kelle und Erzberger 2018, S. 303) zu nutzen und die im quantitativen Verfahren gewonnenen Ergebnisse durch qualitative Ergebnisse im Sinne ihrer Komplementarität (ebenda, S. 305) anzureichern und zu bestätigen. Im qualitativ erhobenen Material ließen sich daher eher subjektive Sinnsetzungen und Handlungsorientierungen vor allem mit Blick auf die Methode eduScrum: digital und die Verwendung von ILIAS ableiten, welche aber mit Blick auf das Erkenntnisinteresse dieses Beitrags ausgeklammert wurden.
} 
umformuliert und so angelegt, dass die Studierenden diese in gestuften Skalen bewerten konnten. In Tab. 4 werden Beispielitems exemplarisch dargestellt und die Bewertungsskalen ausgeführt.

Für die Auswertung der Befragungen wurden die Ergebnisse beider Seminare zusammengeführt und im Sinne einer univariaten, deskriptiven Analyse betrachtet. Der Fokus lag hierbei auf der Häufigkeitsverteilung der Angaben (vgl. Diekmann 2018, S. 669). Die Ergebnisse werden in tabellarischer Form mit Fokus auf ausgewertete Antwortmöglichkeiten und in der Gegenüberstellung zwischen Ein- und Ausgangsbefragung in ihrer prozentualen Häufigkeit dargestellt.

Einschränkend muss darauf verwiesen werden, dass die vorliegenden empirischen Daten auf der Selbsteinschätzung der Studierenden basieren und keine Fassung des tatsächlichen Kompetenzzuwachses in Bezug auf die Methode vorgenommen werden kann. Zudem gab es keine kontrastierende Kontrollgruppe, sondern die gleichen Personengruppen wurden vor und nach ihrer Teilnahme an den mit eduScrum: digital umgesetzten Seminaren befragt. 


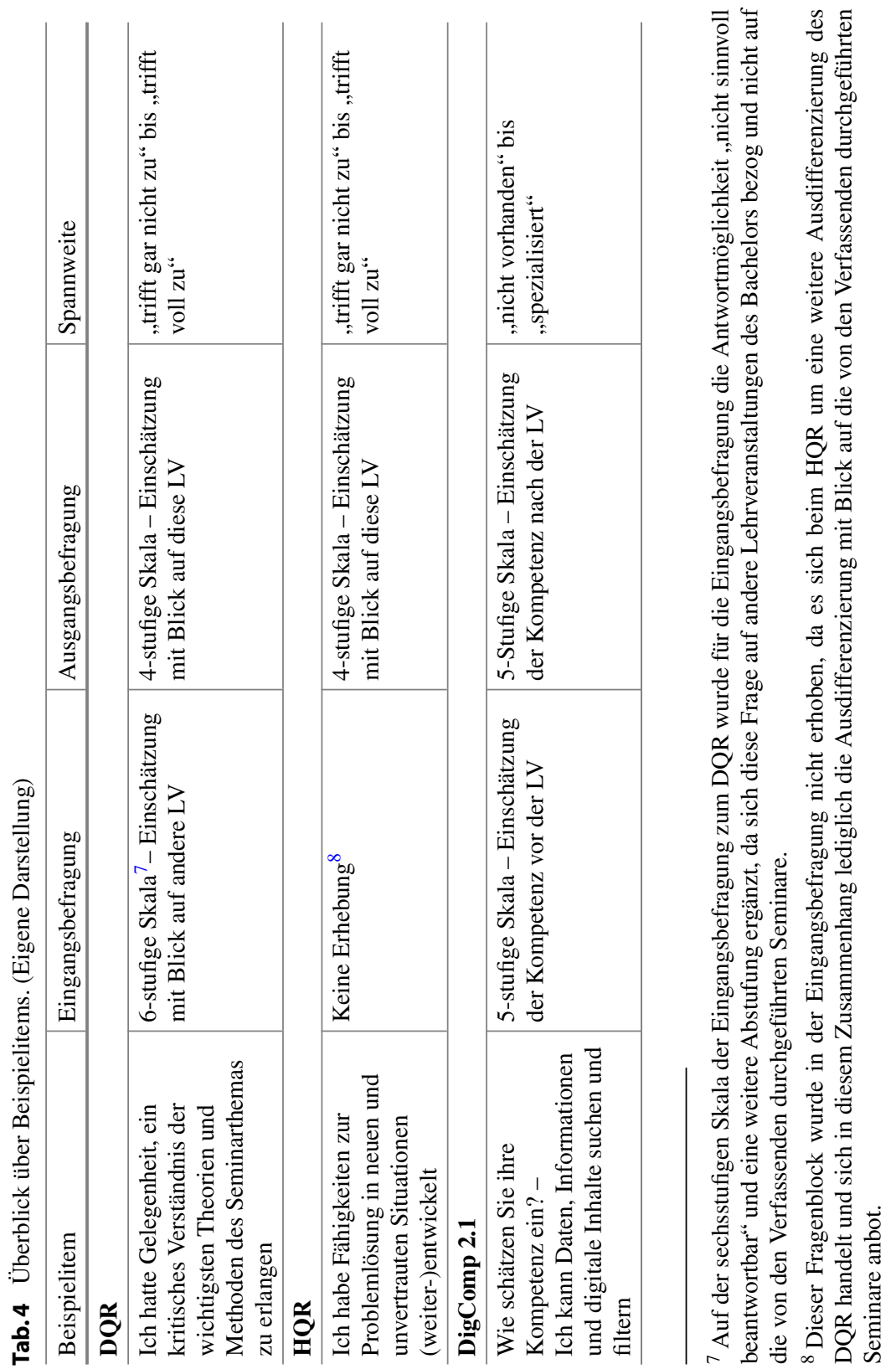




\section{5 eduScrum: digital umgesetzt - erste Ergebnisse und Fazit}

Unter Rückgriff auf die ausgewählten (studienfachunabhängigen) Kompetenzbereiche der zugrunde liegenden Referenzrahmen (DQR/HQR/DigComp 2.1) werden im Folgenden die Ergebnisse präsentiert und bewertet. Abschließend wird unter Rückbindung auf die Ausgangsfrage eine Einschätzung vorgenommen, inwiefern die digital angereicherte Methode dazu geeignet ist, um gezielt - aus dem Referenzrahmen abgeleitete - Kompetenzen zu entwickeln.

\subsection{Ergebnisse in Bezug auf den DQR/HQR}

In Anlehnung an die ausgewählten Kompetenzen des DQR 6 zeigt Tab. 5, wie die Studierenden ihren eigenen Kompetenzzuwachs in Bezug auf das Seminar beurteilen. Spalte 1 zeigt die an den DQR-Kompetenzen orientierten Aussagen zur Selbsteinschätzung aus der Ausgangsbefragung. Die zweite Spalte zeigt die Zustimmung der Studierenden zu diesen Aussagen in Anlehnung an das durchgeführte Seminar (hier wurden die Kategorien ,trifft eher zu“ und „trifft voll zu“ zusammengefasst). Spalte 3 greift auf Daten der Eingangsbefragung zurück. Die Studierenden wurden gefragt, inwiefern der Erwerb der genannten Kompetenzen ihrer Einschätzung nach in Lehrveranstaltungen, die sie bereits im hochschulischen Kontext besucht haben, gezielt gefördert wurde (hier wurden die Kategorien „trifft für den überwiegenden Teil der Lehrveranstaltungen“ und „trifft für nahezu alle Lehrveranstaltungen zu“" zusammengefasst).

Zum einen weisen die Daten darauf hin, dass sich eduScrum: digital nach Einschätzung der Studierenden gut eignet, um studienfachunabhängig die im DQR definierten Kompetenzen zu entwickeln. Die Studierenden bewerten den Beitrag der Methode für die Entwicklung ihrer Kompetenzen über alle drei Kategorien hinweg (Fertigkeiten, Sozialkompetenz und Selbstständigkeit) als hoch. Zum anderen geben die Studierenden an, dass die Lehrveranstaltungen, an denen sie bislang im hochschulischen Kontext teilgenommen haben, diese Kompetenzen eher weniger gezielt fördern oder möglicherweise nicht so explizit thematisieren wie diese Seminare. Insgesamt weisen die gewonnenen Erkenntnisse darauf hin, dass in Bezug auf die im DQR beschriebenen überfachlichen Kompetenzen eine gezielte Entwicklung von fachlichen und personalen Kompetenzen im Sinne der Lernziele des Studienprogramms erfolgt ist.

In Anlehnung an die nach dem HQR auf Bachelorniveau zu vermittelnden Kompetenzen wurden die Studierenden im Rahmen der Ausgangsbefragung 
Tab. 5 Selbsteinschätzung des Kompetenzzuwachses in Bezug auf den DQR. (Eigene Darstellung)

\begin{tabular}{l|l|l}
\hline & $\begin{array}{l}\text { Kompetenzentwicklung im } \\
\text { Seminar (\%) }\end{array}$ & $\begin{array}{l}\text { Kompetenzentwicklung in } \\
\text { anderen Seminaren (\%) }\end{array}$ \\
\hline
\end{tabular}

\section{Fachkompetenz}

\section{Fertigkeiten}

\begin{tabular}{l|l|l}
\hline $\begin{array}{l}\text { Ich konnte mir ein kritisches } \\
\text { Verständnis der wichtigsten } \\
\text { Theorien und Methoden des } \\
\text { wissenschaftlichen Fachs } \\
\text { erarbeiten }\end{array}$ & 87,72 & 56,52 \\
\hline $\begin{array}{l}\text { Ich kann neue Lösungen } \\
\text { erarbeiten und diese unter } \\
\text { Berücksichtigung } \\
\text { unterschiedlicher Maßstäbe } \\
\text { beurteilen, auch bei sich } \\
\text { häufig ändernden } \\
\text { Anforderungen }\end{array}$ & 85,97 & 27,54 \\
\hline
\end{tabular}

\section{Personale Kompetenz}

\section{Sozialkompetenz}

\begin{tabular}{l|l|l}
\hline $\begin{array}{l}\text { Ich bin in der Lage, mit } \\
\text { anderen Studierenden in } \\
\text { Teams eigenverantwortlich } \\
\text { zusammenzuarbeiten }\end{array}$ & 96,49 & 62,32 \\
\hline $\begin{array}{l}\text { Ich kann vorausschauend mit } \\
\text { Problemen im Team } \\
\text { umgehen }\end{array}$ & 91,23 & 31,88 \\
\hline $\begin{array}{l}\text { Ich verfüge über die } \\
\text { Kompetenz, komplexe } \\
\text { fachbezogene Probleme zu } \\
\text { lösen und meine Ergebnisse } \\
\text { argumentativ zu vertreten }\end{array}$ & 94,74 & 31,87 \\
\hline
\end{tabular}

\section{Selbstständigkeit}

\begin{tabular}{l|l|l}
\hline $\begin{array}{l}\text { Ich wurde befähigt, Ziele für } \\
\text { Lehr- und Lernprozesse zu } \\
\text { definieren, zu reflektieren } \\
\text { und zu bewerten }\end{array}$ & 91,23 & 30,43 \\
\hline $\begin{array}{l}\text { Ich kann Lern- und } \\
\text { Arbeitsprozesse eigenständig } \\
\text { und nachhaltig gestalten }\end{array}$ & 98,25 & 53,61 \\
\hline
\end{tabular}


danach gefragt, inwiefern die Umsetzung in den Seminaren dazu beigetragen hat, die geforderten Kompetenzen im (studienfachunabhängigen) Bereich der Wissenserschließung zu entwickeln. Tab. 6 zeigt in der linken Spalte die aus dem HQR entnommenen Kompetenzen und in der rechten Spalte die Zustimmungswerte der Studierenden (dabei wurden die Antwortmöglichkeiten ,trifft eher zu“ und ,trifft voll zu“ zusammengefasst).

Auch vor der Reflexionsfolie der Anforderungen des HQR bewerten die Studierenden die Methode eduScrum: digital in der Breite als hilfreich, um die festgeschriebenen Kompetenzen zu entwickeln. Insbesondere systemische Kompetenzen wie das selbstständige Aneignen von Wissen oder das Lösen komplexer Probleme, aber auch kommunikative Kompetenzen wie die eigene Vermittlungskompetenz werden - nach (Selbst-)Einschätzung der befragten Studierenden - durch die Methode gefördert.

Zusammenfassend kann resümiert werden, dass die Umsetzung der Methode nach Einschätzung der Studierenden sehr gut geeignet erscheint, um sowohl die Fachkompetenzen und Sozialkompetenzen (DQR) als auch den Bereich der Wissenserschließung (HQR) zu fördern.

\subsection{Ergebnisse in Bezug auf den DigComp 2.1}

In Anlehnung an die ausgewählten Kompetenzen des DigComp 2.1 zeigt die untenstehende Tab. 7, wie viele der Studierenden ihre Kompetenzen vor und nach dem Seminar auf den Leveln Fortgeschritten und Spezialisiert (Stufen 5/6) eingeschätzt haben. Die erste Spalte zeigt die Aussagen, die aus den Kompetenzbereichen erstellt wurden. In den Spalten 2 und 3 werden die beiden Qualifikationslevel auf Bachelorniveau abgebildet, wobei sich die Ergebnisse aus der Eingangsbefragung (VOR) und Ausgangsbefragung (NACH) generieren. 
Tab. 6 Selbsteinschätzung des Kompetenzzuwachses in Bezug auf den HQR. (Eigene Darstellung)

\begin{tabular}{l|l}
\hline & Kompetenzentwicklung im Seminar (\%) \\
\hline Können (Wissenserschließung) & \\
\hline $\begin{array}{l}\text { Instrumentale Kompetenzen } \\
\begin{array}{l}\text { Ich habe Fähigkeiten zur Problemlösung in } \\
\text { neuen und unvertrauten Situationen } \\
\text { (weiter)entwickelt }\end{array}\end{array}$ & 87,72 \\
\hline
\end{tabular}

\section{Systemische Kompetenzen}

Ich habe zur Lösung komplexer Aufgaben beigetragen

Ich habe auf der Grundlage unvollständiger 94,74 oder begrenzter Informationen wissenschaftlich fundierte Entscheidungen gefällt

Ich habe mir selbstständig neues Wissen und Können angeeignet

\begin{tabular}{l|l}
\hline Ich habe weitgehend selbst gesteuert und/oder & 82,45
\end{tabular}
autonom eigenständige forschungs- oder anwendungsorientierte Projekte durchgeführt

\section{Kommunikative Kompetenzen}

\begin{tabular}{l|l}
\hline $\begin{array}{l}\text { Ich habe weitgehend selbst gesteuert und/oder } \\
\text { autonom eigenständige forschungs- oder } \\
\text { anwendungsorientierte Projekte durchgeführt }\end{array}$ & 82,45 \\
\hline $\begin{array}{l}\text { Ich habe auf dem aktuellen Stand der Forschung } \\
\text { und Anwendung Fachvertreter*innen und Laien } \\
\text { meine Schlussfolgerungen vermittelt }\end{array}$ & 78,95 \\
\hline $\begin{array}{l}\text { Die obigen Schlussfolgerungen habe ich } \\
\text { mithilfe zugrunde liegender Informationen und }\end{array}$ & 91,23 \\
$\begin{array}{l}\text { Beweggründe in klarer und eindeutiger Weise } \\
\text { vermittelt }\end{array}$ & \\
\hline $\begin{array}{l}\text { Ich habe mich mit Fachvertreter*innen und mit } \\
\text { Laien über Informationen, Ideen, Probleme und }\end{array}$ & 71,93 \\
$\begin{array}{l}\text { Lösungen auf wissenschaftlichem Niveau } \\
\text { ausgetauscht }\end{array}$ & \\
\hline $\begin{array}{l}\text { Ich habe in meinem Team herausgehobene } \\
\text { Verantwortung übernommen }\end{array}$ & 73,96 \\
\hline
\end{tabular}


Tab. 7 Selbsteinschätzung des Kompetenzzuwachses in Bezug auf DigComp 2.1. (Eigene Darstellung)

\begin{tabular}{l|l|l|l|l}
\hline & \multicolumn{2}{|l|}{ Fortgeschritten } & \multicolumn{2}{l}{ Spezialisiert } \\
\hline $\begin{array}{l}\text { Informations- und } \\
\text { Datenkompetenz }\end{array}$ & VOR (\%) & NACH (\%) & VOR (\%) & NACH (\%) \\
\hline $\begin{array}{l}\text { Ich kann Daten, Informationen } \\
\text { und digitale Inhalte suchen } \\
\text { und filtern }\end{array}$ & 49,13 & 52,64 & 8,77 & 21,05 \\
\hline $\begin{array}{l}\text { Ich kann Daten, Informationen } \\
\text { und digitale Inhalte } \\
\text { einschätzen und bewerten }\end{array}$ & 42,11 & 61,41 & 12,28 & 15,79 \\
\hline $\begin{array}{l}\text { Ich kann Daten, Informationen } \\
\text { und digitale Inhalte verwalten }\end{array}$ & 42,11 & 49,13 & 15,75 & 21,05 \\
\hline
\end{tabular}

\section{Kommunikation und Kollaboration}

\begin{tabular}{|c|c|c|c|c|}
\hline $\begin{array}{l}\text { Ich kann mit anderen durch } \\
\text { digitale Technologien } \\
\text { interagieren }\end{array}$ & 49,13 & 50,88 & 15,79 & 29,82 \\
\hline $\begin{array}{l}\text { Ich kann Inhalte mithilfe von } \\
\text { digitalen Technologien teilen }\end{array}$ & 45,61 & 49,13 & 22,81 & 28,07 \\
\hline $\begin{array}{l}\text { Ich kann mithilfe von digitalen } \\
\text { Technologien Verantwortung } \\
\text { übernehmen }\end{array}$ & 29,82 & 49,13 & 5,26 & 12,28 \\
\hline $\begin{array}{l}\text { Ich kann mithilfe digitaler } \\
\text { Technologien mit anderen } \\
\text { zusammenarbeiten }\end{array}$ & 45,62 & 49,13 & 21,05 & 33,33 \\
\hline $\begin{array}{l}\text { Ich kann } \\
\text { Kommunikationsstandards } \\
\text { (Nettiquette) anwenden }\end{array}$ & 36,85 & 36,85 & 15,79 & 15,79 \\
\hline $\begin{array}{l}\text { Ich kann digitale Identitäten } \\
\text { verwalten }\end{array}$ & 33,33 & 26,31 & 3,51 & 12,28 \\
\hline \multicolumn{5}{|l|}{ Digitale Inhalte erstellen } \\
\hline $\begin{array}{l}\text { Ich kann digitale Inhalte } \\
\text { entwickeln }\end{array}$ & 36,85 & 43,86 & 5,26 & 17,54 \\
\hline $\begin{array}{l}\text { Ich kann digitale Inhalte neu } \\
\text { bearbeiten und einbinden }\end{array}$ & 45,36 & 54,38 & 8,77 & 15,79 \\
\hline $\begin{array}{l}\text { Ich kann Urheberrecht und } \\
\text { Lizenzrecht anwenden }\end{array}$ & 10,53 & 19,30 & 8,77 & 7,02 \\
\hline Ich kann programmieren & 0,00 & 3,51 & 0,00 & 0,00 \\
\hline
\end{tabular}


Aus den Ergebnissen wird zum einen deutlich, dass sich über die Hälfte der Studierenden (in nahezu allen Fällen) - außer in Bezug auf die Verantwortungsübernahme, Urheberrecht und Programmieren - bereits auf einem hohen Level verortet. Zum anderen zeigt sich, dass die Studierenden sich nach dem Seminar in fast allen Bereichen stärker einschätzen, als sie es vor dem Seminar getan haben. Dies gilt sowohl für Studierende, die mit einer höheren Selbsteinschätzung in das Seminar eingestiegen sind, als auch für Studierende, die sich zu Beginn schwächer eingeschätzt haben. Hieraus lassen sich zwei zentrale Erkenntnisse ableiten:

Auf der einen Seite zeigt sich, dass sich nicht alle Studierenden im Bereich der digitalen Kompetenzen auf der - dem Bachelorniveau entsprechenden - Stufe des Referenzrahmens verorten, insbesondere nicht in rechtlichen Aspekten und in Bezug auf Programmieren. Auf der anderen Seite wird deutlich, dass eduScrum: digital in den Seminaren nach Einschätzung der Studierenden einen positiven Effekt auf die Förderung ihrer digitalen Kompetenzen hat. ${ }^{9}$ Insbesondere beim Verwenden und Anwenden von digitalen Tools - vor allem auch im Bereich des Erstellens, Bearbeitens und Teilens von Inhalten - und der Notwendigkeit der Zusammenarbeit und Kommunikation in den Teams lassen sich positive Entwicklungen beobachten.

\section{$6 \quad$ Fazit und Ausblick}

Die Ergebnisse liefern vielfältige Hinweise darauf, dass sich die Methode dazu eignet, auf der einen Seite gezielt die Entwicklung von fachlichen und personalen Kompetenzen sowie Kompetenzen auf dem Feld der Wissenserschließung im Sinne der Lernziele eines Studienprogramms zu fördern. Auf der anderen Seite katalysiert die Methode gleichzeitig die Entwicklung von Kompetenzen im Umgang mit digitalen Tools, sowohl zur Erstellung von Inhalten als auch im Kontext des kooperativen Arbeitens. Vor dem Hintergrund der zur Verfügung stehenden Daten eignet sich die Methode eduScrum: digital, um studienfachunabhängig Studierende dabei zu unterstützen, die von den einschlägigen Referenzrahmen definierten Kompetenzen auf Bachelorniveau zu entwickeln. Um die im Rahmen dieses Beitrages vorgestellten Ergebnisse weiter anreichern und besser einordnen zu können, werden die Verfassenden diese zudem in einem nächsten Schritt mit den Ergebnissen der qualitativen Erhebungen verschränken.

\footnotetext{
${ }^{9}$ Die Felder Programmieren und Urheberrecht standen in diesem Durchgang des Seminars nicht explizit im Fokus der Umsetzung, könnten aber theoretisch ebenso mitaufgenommen werden. Für die Fragen von Urheberrecht in der Erstellung von Materialien ist dies inhaltlich schon in die Planung der nächsten Umsetzung aufgenommen.
} 
Relativierend muss angemerkt werden, dass im Rahmen der hier vorgenommenen explorativen Studie lediglich die Selbsteinschätzung von Studierenden erhoben wurde. Darüber hinaus ist zu berücksichtigen, dass keine Daten einer Kontrollgruppe erhoben wurden und dass nicht ausgeschlossen werden kann, dass es bei der Beantwortung der Fragen (trotz absoluter Anonymität) einen Effekt der sozialen Erwünschtheit gab.

Weiterführend könnte daher eine objektive Messung von tatsächlich vorhandenen Kompetenzen vor und nach den Seminaren im Verhältnis zu einer kontrastierenden Kontrollgruppe vorgenommen werden. Darüber hinaus sollten die Ergebnisse der Studie aufgrund der relativ geringen Probandenzahl ( $\mathrm{n}=$ 66) in einer Untersuchung mit einer größeren Stichprobe bestätigt werden. Die eindeutig positive Beantwortung der Ausgangsfrage liefert jedoch aus Sicht der Verfassenden einen begründeten Anlass für weiterführende und tiefergehende Betrachtungen, um die hier präsentierten Befunde in breiter angelegten Untersuchungen $\mathrm{zu}$ überprüfen und weiter zu differenzieren.

\section{Literatur}

Arbeitskreis Deutscher Qualifikationsrahmen. (2011). Deutscher Qualifikationsrahmen für lebenslanges Lernen. https://www.dqr.de/media/content/Der_Deutsche_Qualifikationsr ahmen_fue_lebenslanges_Lernen.pdf. Zugegriffen: 14. Mai 2020.

Carretero, S., Vuorikari, R., \& Punie, Y. (2017). DigComp 2.1: The digital competence framework for citizens with eight proficiency levels and examples of use. EUR 28558 EN. https://doi.org/10.2760/38842.

Delhij, A., van Solingen, R., \& Wijnands, W. (2015). Der eduScrum Guide. „Die Spielregeln“. https://eduscrum.nl/en/file/CKFiles/Der_eduScrum_Guide_DE_1.2.pdf. Zugegriffen: 14. Mai 2020.

Diekmann, A. (2018). Empirische Sozialforschung. Reinbek bei Hamburg: Rowohlt.

Ferrari, A., Punie, Y., \& Brecko, B. (2013). DIGCOMP: A framework for developing and understanding digital competence in Europe. https://publications.jrc.ec.europa.eu/reposi tory/bitstream/JRC83167/lb-na-26035-enn.pdf. Zugegriffen: 15. Okt. 2020.

Ferrari, A., Brecko, B., \& Punie, Y. (2014). DIGCOMP: A framework for developing and understanding digital comnetence in Europe. eLearning papers, 38, 1-14.

Hochschulrektorenkonferenz, Kultusministerkonferenz. (2017). Qualifikationsrahmen für deutsche Hochschulabschlüsse. https://www.dqr.de/media/content/HQR_Stand_16.02. 2017.pdf. Zugegriffen: 14. Mai 2020.

Kelle, U., \& Erzberger, C. (2018). Qualitative und quantitative Methoden: kein Gegensatz. In U. Flick, E. v. Kardorff, \& I. Steinke (Hrsg.), Qualitative Forschung (S. 299-308). Reinbek bei Hamburg: Rowohlt.

Meyer-Gunkel, V., Klier, J., Kirchherr, J., \& Winde, M. (2019). Future Skills: Strategische Potenziale für Hochschulen. Future Skills Diskussionspapier, 3. Essen: Stifterverband für 
die Deutsche Wissenschaft e. V. https://www.stifterverband.org/download/file/fid/7213. Zugegriffen: 14. Mai 2020.

Metzner, J., Bartosch, U., Vogel, M., Schroll, A.-L., Rademacher, M., \& Neuhausen, H. (2019). Was bedeutet Hochschullehre im digitalen Zeitalter? Eine Betrachtung des Bildungsbegriffs vor den Herausforderungen der Digitalisierung (Arbeitspapier, 50). Berlin: Hochschulforum Digitalisierung. https://doi.org/10.5281/zenodo.4282368.

Schwaber, K., \& Sutherland, J. (2017). Der Scrum Guide. Der gültige Leitfaden für Scrum: Die Spielregeln. https://www.scrumguides.org/docs/scrumguide/v2017/2017Scrum-Guide-German.pdf. Zugegriffen: 14. Mai 2020.

Sturm, N., \& Rundnagel, H. (2020). Agiles Lehren und Lernen an Hochschulen - Potenziale von eduScrum. In B. Berendt, A. Fleischmann, N. Schaper, B. Szczyrba, \& J. Wildt (Hrsg.), Neues Handbuch für Hochschullehre. Berlin: DUZ.

Vuorikari, R., Punie, Y., Carretero Gomez S., \& Van den Brande, G. (2016). DigComp 2.0: The digital competence framework for citizens. Update phase 1: The conceptual reference model. EUR 27948 EN. Luxemburg: Publication Office of the European Union. https:// doi.org/10.2791/11517.

Wijnands, W., \& Stolze, A. (2019). Transforming education with eduScrum. In D. Parsons \& K. McCallum (Hrsg.), Agile and lean concepts: Bringing methodologies from industry to the classroom (S. 95-114). Singapore: Springer.

Open Access Dieses Kapitel wird unter der Creative Commons Namensnennung 4.0 International Lizenz (http://creativecommons.org/licenses/by/4.0/deed.de) veröffentlicht, welche die Nutzung, Vervielfältigung, Bearbeitung, Verbreitung und Wiedergabe in jeglichem Medium und Format erlaubt, sofern Sie den/die ursprünglichen Autor(en) und die Quelle ordnungsgemäß nennen, einen Link zur Creative Commons Lizenz beifügen und angeben, ob Änderungen vorgenommen wurden.

Die in diesem Kapitel enthaltenen Bilder und sonstiges Drittmaterial unterliegen ebenfalls der genannten Creative Commons Lizenz, sofern sich aus der Abbildungslegende nichts anderes ergibt. Sofern das betreffende Material nicht unter der genannten Creative Commons Lizenz steht und die betreffende Handlung nicht nach gesetzlichen Vorschriften erlaubt ist, ist für die oben aufgeführten Weiterverwendungen des Materials die Einwilligung des jeweiligen Rechteinhabers einzuholen.

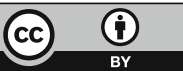

